\title{
Chimera states in small optomechanical arrays
}

\author{
Karl Pelka $\odot,{ }^{1, *}$ Vittorio Peano $\odot,{ }^{2,1}$ and André Xuereb $\odot^{1}$ \\ ${ }^{1}$ Department of Physics, University of Malta, Msida MSD 2080, Malta \\ ${ }^{2}$ Max Planck Institute for the Science of Light, Staudtstraße 2, 91058 Erlangen, Germany
}

(Received 20 June 2019; accepted 14 January 2020; published 25 February 2020)

\begin{abstract}
Synchronization of weakly coupled nonlinear oscillators is a ubiquitous phenomenon that has been observed across the natural sciences. We study the dynamics of optomechanical arrays-networks of mechanically compliant structures that interact with the radiation pressure force-which are driven to self-oscillation. These systems offer a convenient platform to study synchronization phenomena and have potential technological applications. We demonstrate that this system supports the existence of long-lived chimera states, where parts of the array synchronize while others do not. Through a combined numerical and analytical analysis we show that these chimera states can only emerge in the presence of mechanical frequency heterogeneity.
\end{abstract}

DOI: 10.1103/PhysRevResearch.2.013201

\section{INTRODUCTION}

The synchronization of weakly coupled oscillators is a common feature of nonlinear dynamics that arises in various disciplines ranging from engineering to neuroscience [1]. The paradigmatic Kuramoto model [2] explains how an ensemble of phase oscillators can exhibit collective synchronization induced by identical all-to-all coupling, in spite of differences in their natural frequencies. Aside from realizations in biological systems [3,4], synchronization of coupled oscillators finds technological applications, e.g., in high-power laser diode arrays having high efficiency and low divergence [5-7].

Kuramoto [8] discovered that the same type of nonlinear interaction can lead to emergent phenomena for identical phase oscillators upon relaxing from a global identical coupling to a nonlocal coupling topology. These arrangements can be used to implement finite state machines [9], for example. However, they are also known to fail to synchronize completely, but rather to support coexistence of coherence and incoherence-later dubbed chimera states [10]—under specific conditions that are still being investigated. While the Kuramoto model is known to be analytically reducible with the Watanabe-Strogatz ansatz [11], further results on generalized Kuramoto models with finite degrees of freedom or in the continuum limit gave insight into the relevant order parameters and their evolution in time [12-15]. Additional analyses were conducted showing that chimera states can be transient [16] or stable [17,18], as well as robust to heterogeneities in natural frequencies [19-21], coupling topologies [22,23], and noise $[24,25]$. Theoretical work [26,27] also motivated successful experimental observations of such states in arrays

\footnotetext{
*karl.a.pelka@um.edu.mt

Published by the American Physical Society under the terms of the Creative Commons Attribution 4.0 International license. Further distribution of this work must maintain attribution to the author(s) and the published article's title, journal citation, and DOI.
}

of coupled chemical oscillators [28-30], spatial light modulators [31], optoelectronic networks [32], and metronomes on swings [33]. Of contemporary interest in the study of network dynamics are the effects of phase interactions that include higher harmonics, as well as next-to-nearest-neighbor and triadic interactions in theory and experiment [34].

The study of the dynamics of micromechanical systems has undergone tremendous growth in recent years under the guise of optomechanics [35]. The prototypical optomechanical system consists of a single mode of the electromagnetic radiation field, e.g., within a high-finesse optical cavity [36], interacting with the motion of a harmonic oscillator by means of the radiation pressure force [37]. The moving element variously takes the form of one of the end mirrors of a cavity [38], a semi-transparent membrane inside a cavity [39], one plate of a capacitor [40], a micro- or nano-particle in a cavity [41,42], or the cavity itself in the case of micro-toroids supporting whispering gallery modes of the radiation field [36]. Various experimental techniques make these systems tunable in their optical [43] and mechanical properties [44]. The optomechanical interaction has been used to cool the motion of the mechanical system down to its ground state $[40,45]$, generate quantum entanglement between mechanical oscillators [46,47], and produce proof-of-concept isolators and directional amplifiers for microwave radiation [48-51]. Recent work has started exploring the many-body dynamics of systems of coupled optomechanical networks, including the possibility of obtaining stronger coupling at the singlephoton level [52,53], topological physics [54-56], dynamical gauge fields [57], and synchronization phenomena [58-66]. Such systems may also find technological use; synchronized optomechanical arrays, for example, could act as high-power and low-noise on-chip frequency sources [65].

Synchronization is especially interesting as a many-body phenomenon since it requires only a few optomechanical cells and its experimental observation $[65,66]$ raises the question of its robustness against heterogeneity in the natural frequencies of the mechanical oscillators, as well as potential interactions of multiple arrays on one chip. Only recently, chimera states 
were hypothesized to arise in optomechanical arrays [66]. To explore this question we employ a model of two arrays of optomechanical oscillators subject to global mechanical coupling within each as well as with the other array. Our theoretical analysis reveals that chimera states arise in two indistinguishable arrays, each composed of as few as four oscillators, in contrast to other collective effects [54-57] that require very large numbers of oscillators. Furthermore, our work identifies limitations for the large-scale integration of optomechanical arrays on a chip.

Following the introduction of our model, we describe the results of exhaustive numerical experiments that identify the region of parameter space where chimera states arise in our model. We then analyze the continuum limit to obtain analytical results, and conclude by discussing the implications of our results for applications of optomechanical arrays.

\section{MODEL}

We consider the collective dynamics of two identical optomechanical arrays, $\theta=1,2$, each of which consists of $N$ mechanical modes coupled to one global laser-driven optical mode of amplitude $\alpha^{\theta}$, which is described by the equations of motion $[58,64,65]$

$$
\begin{gathered}
m\left(\ddot{x}_{i}^{\theta}+\Gamma \dot{x}_{i}^{\theta}+\Omega_{i}^{\theta 2} x_{i}^{\theta}\right)=F_{i, \mathrm{opt}}^{\theta}(t)+\sum_{\theta^{\prime}=1,2} F_{i, \mathrm{~m}}^{\theta \theta^{\prime}}, \text { and } \\
\dot{\alpha}^{\theta}=\left[i\left(\Delta-\delta \omega_{\mathrm{opt}}\right)-\frac{\kappa}{2}\right] \alpha^{\theta}+\frac{\kappa}{2} \alpha_{\max } .
\end{gathered}
$$

Here, $x_{j}^{\theta}, \Omega_{i}^{\theta}, m$, and $\Gamma$ denote the displacement, natural frequency, effective mass, and damping rate of the mechanical modes. We assume that the arrays contain identical sets of oscillators, i.e., $\Omega_{i}^{1}=\Omega_{i}^{2}=: \Omega_{i}$ such that the two arrays are indistinguishable. Each optical mode is characterized by its decay rate $\kappa$ and its detuning from the driving laser $\Delta=$ $\omega_{\text {laser }}-\omega_{\text {opt }}$, which we assume are independent of $\theta$. The optomechanical interaction shifts the resonance frequency by $\delta \omega_{\mathrm{opt}}=-\sum G x_{j}^{\theta}$ as a result of the mechanical displacements and imparts a force $F_{i, \mathrm{opt}}^{\theta}=\hbar G\left|\alpha^{\theta}\right|^{2}$ on the mechanical modes. For blue detuning $(\Delta>0)$ and large-enough optical power, there exists a Hopf bifurcation leading to synchronized selfoscillation $[37,58,65]$.

The focus of this work is the analysis of additional mechanical coupling between the arrays and its effects on their synchronization. The mechanical coupling $F_{i, \mathrm{~m}}^{\theta \theta^{\prime}}=\sum_{j} k_{i j}^{\theta \theta^{\prime}}\left(x_{j}^{\theta^{\prime}}-\right.$ $\left.x_{i}^{\theta}\right)$ is assumed to be global: $k_{i j}^{\theta \theta^{\prime}}=\left(1-\delta_{i j} \delta_{\theta \theta^{\prime}}\right) \mu / N$, i.e., every pair of oscillators is coupled with strength $\mu / N$. Such global springlike coupling was shown theoretically [58] and realized experimentally for two oscillators [64] with an optomechanical coupling driven with a red-detuned laser $(\Delta<0)$. We assume the mechanical parameters $m, \Gamma$, and $G$ to be identical to retain the symmetry of the arrays; chimera states are a result of spontaneous symmetry breaking in cases where the oscillator populations are identical. Additionally, we have verified that our numerical results still hold for small variations of these parameters (for details, see Appendix A).

Figure 1 presents a schematic illustration of a realization of this model, i.e., a system consisting of two arrays of microtoroidal optomechanical systems that allows for optical
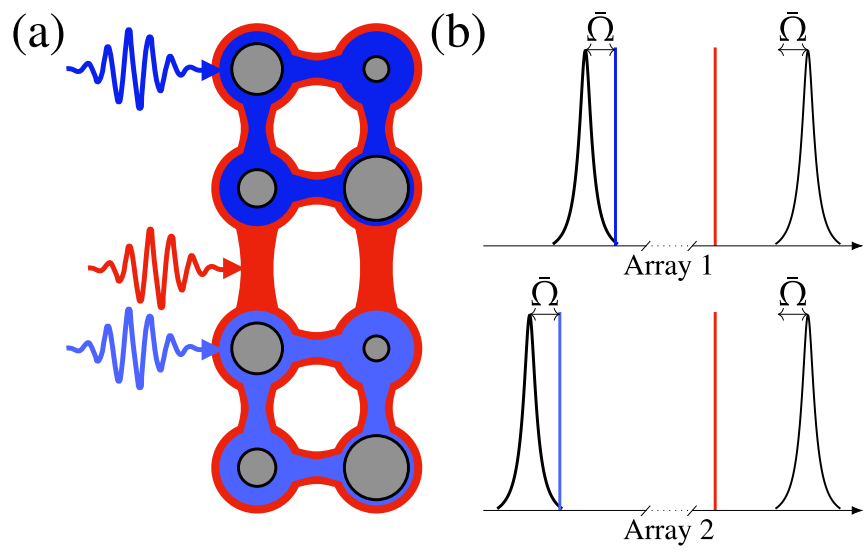

FIG. 1. A test-bed for investigating chimera states. (a) Optomechanical microtoroids are arranged in two identical arrays. Excitation of optical modes delocalized over each array causes self-sustained, synchronized mechanical oscillation at large optical powers. A third optical mode introduces springlike coupling between both arrays allowing chimeras to emerge in the compound system. (b) Driving scheme, where each optical mode is driven coherently with a specific detuning. Further detail is given in the text.

modes delocalized [65] over either one array or both arrays. Driving each array with an optical mode delocalized over it with blue detuning allows the excitation of the mechanical oscillators and self-sustained synchronized oscillation at large powers. Driving another optical mode delocalized over both arrays with red detuning introduces global springlike coupling adjustable via the input power and the detuning.

The behavior of this system depends sensitively on the magnitude of the heterogeneity in the natural frequencies of the mechanical elements, and on the input optical power. An overview of this behavior is depicted in Fig. 2, which is the main numerical result of this work. The statistical evaluation we used to construct Fig. 2 is based on the classification of 60 random initial conditions for the uncoupled system $\mu=0$, varying the maximal photon number $\left|\alpha_{\max }\right|^{2}$ in steps of 500 and the standard deviation $\sigma$ in steps of $0.2 \Gamma$. The natural frequencies we obtained from Fig. S5 of Ref. [65] were 132.1, 132.35, 132.7, and 133.2 MHz. We shifted these to obtain $\bar{\Omega}=133.0 \mathrm{MHz}$ as the mean frequency and rescaled them around $\bar{\Omega}$ to obtain the desired standard deviation $\sigma$, always keeping the mean frequency unchanged. The classification of the initial conditions in the one-array system was performed based on the power spectral densities of the long-time dynamics of the four mechanical oscillators comprising the array. If the respective dominant peaks in the power spectral densities were found to be at different frequencies, the initial condition was classified as not synchronized. If all mechanical frequency peaks coincided at some Fourier frequency $f_{i}$, the initial condition is classified as synchronised to the frequency $f_{i}$. A low-frequency cut-off was imposed at $0.76 \mathrm{MHz}$ due to the presence of numerical noise in this frequency range. For trajectories that exhibited no self-sustained oscillations, the maximum in the power spectral density occured around the cutoff frequency.

This procedure resulted in a probability distribution according to the relative occurrences of each of the 


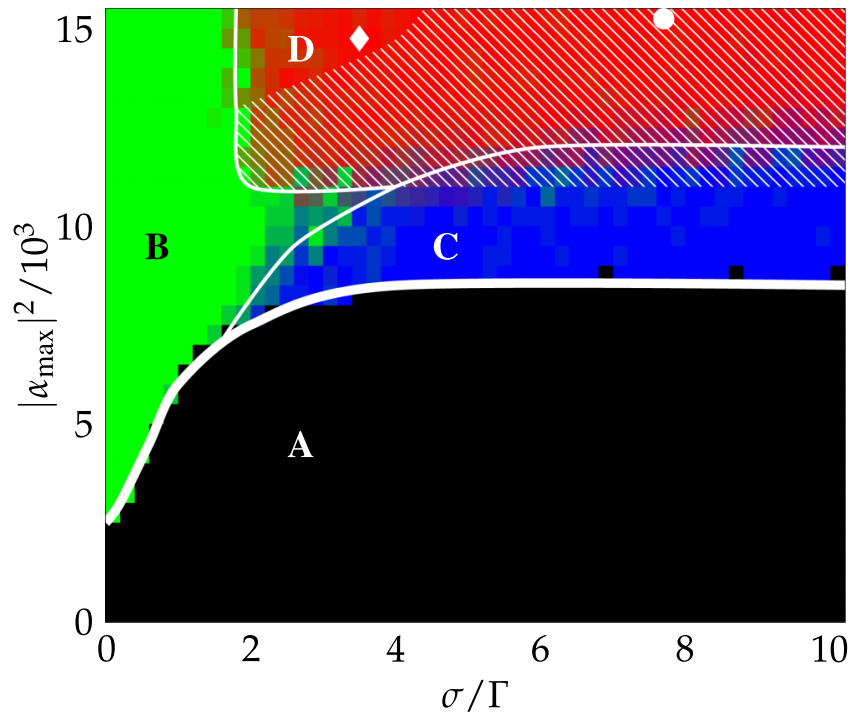

FIG. 2. Two-dimensional parameter-space diagram of the synchronization behavior, plotted as a function of the standard deviation of the natural frequencies, $\sigma$ (horizontal axis), and the maximal number of photons $\left|\alpha_{\max }\right|^{2}$ (vertical). The statistical evaluation process yielding the color of the smooth diagram is detailed in the text. Upon increasing the optical input power $P_{\alpha} \propto\left|\alpha_{\max }\right|^{2}$ distinct behaviors are observed depending on $\sigma$, characterizing the heterogeneity: No selfsustained oscillation (region A, black); self-sustained, synchronized oscillation at one frequency (region B, green); self-sustained, unsychronized oscillation (region $\mathrm{C}$, blue); self-sustained, synchronized oscillation always attained at multiple frequencies (region $\mathrm{D}$, red). This plot can be seen as a two-dimensional projection of a threedimensional parameter space, with the third axis corresponding to the mechanical coupling strength. In the hatched region, additional global mechanical coupling of two arrays leads to chimera states.

above outcomes. For every configuration, the color of the corresponding pixel in Fig. 2 was computed by adopting the following procedure. The blue value is the relative proportion of unsychronized initial conditions. To obtain the red and green values, we computed the entropy $S$ of the relative occurrences $O_{i}$ of the synchronized attractors with respect to the occurrence of synchronized initial conditions $O_{S}: S=$ $-\sum_{i}\left(O_{i} / O_{S}\right) \ln \left(O_{i} / O_{S}\right)$. If there is only one synchronized frequency, all occurrences happen at the same frequency and we have $S=0$. If the occurrences of synchronized oscillation are distributed evenly among all $N$ possible frequencies, we find $S=S_{\max }=\ln (N)$. The green value of each pixel is given by $\left(1-S / S_{\max }\right) O_{S}$ and the red value by $\left(S / S_{\max }\right) O_{S}$. In this scheme, therefore, a green pixel represents a situation where all trajectories synchronized to the same attractor, and a red pixel where trajectories synchronized to all possible attractors with equal probability.

For weak heterogeneity on the scale of a mechanical linewidth, we find that below a threshold input power $P_{\alpha} \propto$ $\left|\alpha_{\text {max }}\right|^{2}$ there is no self-sustained oscillation (region A). Above this threshold, we find synchronized oscillation of all mechanical oscillators for arbitrarily small additional mechanical springlike interaction (region B). The relative phase between the two arrays in the absence of the springlike coupling is arbitrary and depends on the initial condition as required for self- sustained oscillation [1]. In accordance with the analytical insight as discussed below, one finds phase synchronization of the two arrays upon increasing the mechanical interaction.

For large-enough heterogeneity, increasing the optical input power above the oscillation threshold leads to unsynchronized self-sustained oscillation of the arrays (region $\mathrm{C}$ ). Increasing the input power even further (region D), one finds that both arrays always synchronize separately to one of the natural frequencies $\Omega_{i}$ in absence of interaction of the two arrays. Introducing the mechanical interaction between the two arrays can drive one of the arrays out of the synchronized state while the other one is not affected. Since this coexistence of synchronization in one array and lack of sychronization in the other depends crucially on the mechanical coupling between the two arrays, and since the two arrays are identical, we can label these chimera states. Increasing the mechanical interaction between the arrays even further will eventually lead to in-phase or anti-phase synchronization of the two arrays.

\section{NUMERICAL RESULTS WITH SMALL ARRAYS}

Under realistic circumstances, state-of-the-art optomechanical arrays consist of at most a few separate oscillators [65]. Typically, their natural frequencies are spread beyond a linewidth (cf. Fig. S5 in Ref. [65]), but with mechanical tuning procedures [44], one may achieve two indistinguishable arrays experimentally. To address the effect of additional mechanical coupling, we conducted numerical investigations of Eqs. (1) using parameters from Ref. [65]. We consider two identical arrays, each consisting of four oscillators whose natural frequencies are centered around $\bar{\Omega} / 2 \pi=133 \mathrm{MHz}$, and which have a mechanical quality factor $\bar{\Omega} / \Gamma=1000$ and an effective mass $m_{\text {eff }}=70 \mathrm{pg}$. The optical modes interact with each array with a coupling strength $G / 2 \pi=49 \mathrm{MHz} / \mathrm{nm}$ and have a decay rate $\kappa=\bar{\Omega}$. They are driven by a blue-detuned laser with $\Delta=\bar{\Omega}$. The mechanical coupling between the arrays is set to be global; we explore coupling strengths up to $\left|\mu_{\max }\right|=$ $4.1 \times 10^{-3} m \bar{\Omega}^{2}$.

We next turn to examples that illustrate the preceding discussion. Figure 3 shows the behavior of the arrays for the parameters at the white diamond in Fig. 2 with zero (top and middle) and dominant (bottom) mechanical coupling. Without mechanical coupling, collective oscillation of each array takes place at one of the natural frequencies, showing the existence of coherence. Due to the large heterogeneity, the phase difference between pairs of mechanical oscillators will be nonzero. When the mechanical coupling dominates, collective oscillation of the arrays takes place at an arbitrary frequency with the two arrays oscillating in phase, in agreement with the analytical results presented below.

Figure 4 shows sample trajectories of the two arrays for different mechanical coupling strengths with the parameters at the white circle in Fig. 2. The phase reconstructions $\phi_{i}^{\theta}$ are based on the analytical signal representation of the mechanical motion $x_{i}^{\theta}$ (for details, see Appendix B) and their derivatives are represented below as phase velocities. The classification of chimera states is based on the evolution of phase differences $\Delta \phi_{i j}^{\theta}=\phi_{j}^{\theta}-\phi_{i}^{\theta}$ in the respective array. Two oscillators $x_{i}^{\theta}$ and $x_{j}^{\theta}$ are considered mutually synchronized if their phase 


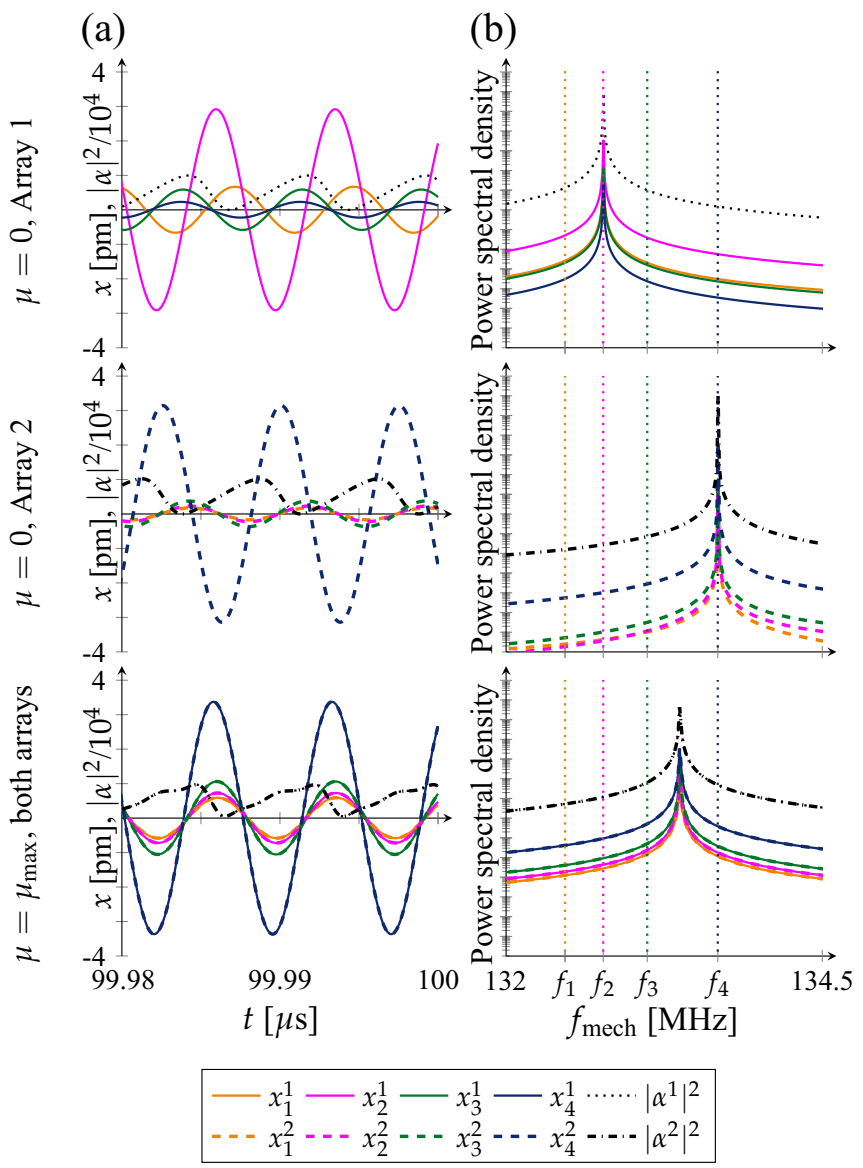

FIG. 3. Numerical analysis of the behavior of two optomechanical arrays consisting of four oscillators and light field each in the absence of (upper and middle row) and with large mechanical coupling (lower row). The chosen parameters correspond to the white diamond in Fig. 2. (a) Time evolution of the mechanical oscillator positions (orange, magenta, green, blue) and light field amplitude (black) (b) Mechanical power spectral densities. In all cases, we find synchronization to one frequency, which in the case of no mechanical coupling is one of the natural frequencies of the oscillators. These natural frequencies are indicated by the vertical lines. In the largecoupling case, both arrays are synchronized in frequency and phase as can be seen by the evolution of the arrays, which are presented on the same plot.

difference $\Delta \phi_{i j}^{\theta}$ in the tested time interval, which is taken to be significantly longer than any characteristic time period, varies less than $2 \pi$, and mutually unsynchronized otherwise. An array is considered synchronized if all oscillators are mutually synchronized and unsynchronized otherwise. A state is considered a chimera state if there is a combination of synchronized initial conditions for each individual array and at least one mechanical coupling $\mu_{\mathrm{Ch}}$ such that one array is synchronized and the other is not, as it is the case for the example illustrated in Fig. 4. The top (bottom) row represents the first (second) array, and the columns represent zero (left), intermediate (middle), and dominant (right) mechanical coupling. For zero mechanical coupling, the two arrays synchronize independently of each other to one of the natural frequencies of their oscillators. This is indicated by all the phases of
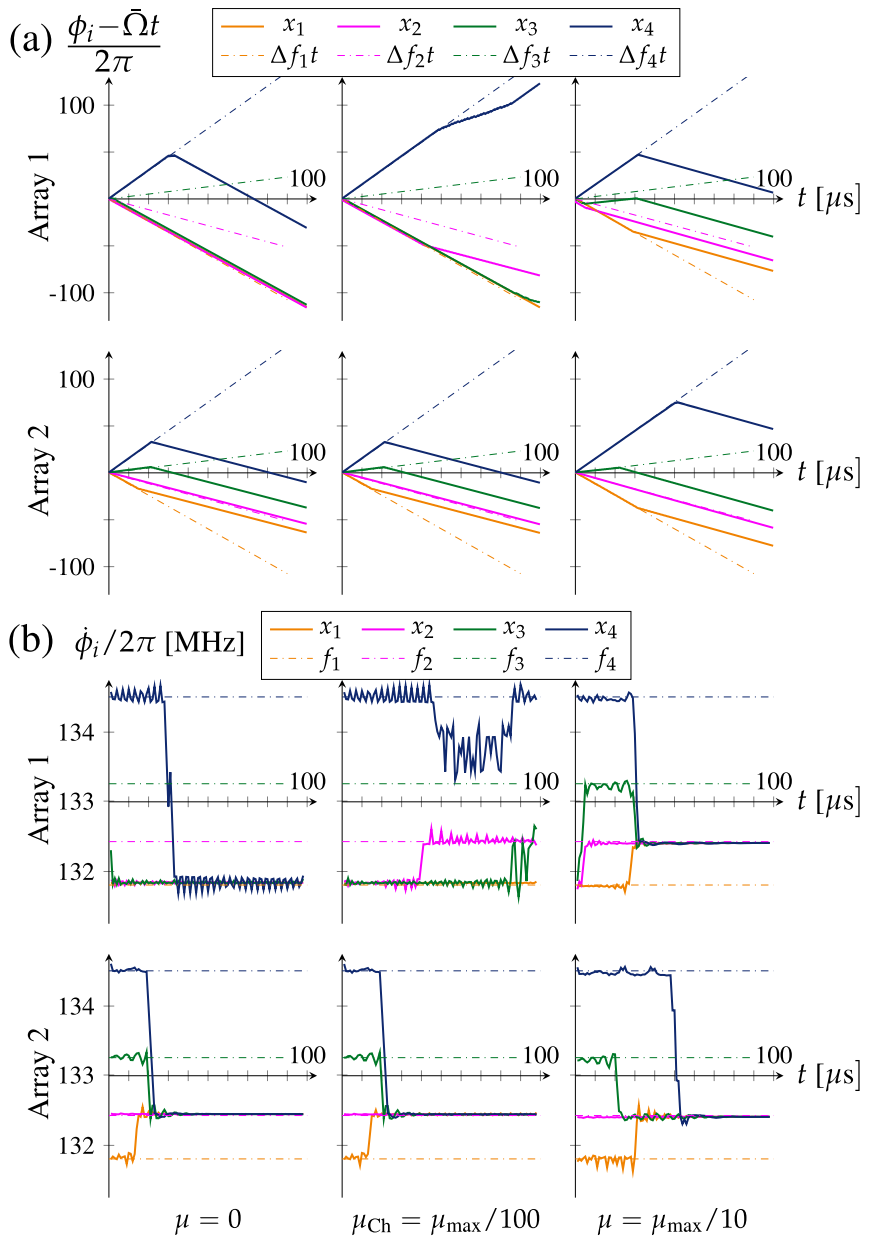

FIG. 4. Sample trajectories of the mechanical motion (orange, magenta, green, blue) for both arrays (white circle in Fig. 2). (a) Phase reconstruction via analytic signal representation and (b) phase velocities extracted from the reconstructed phases. The top (bottom) row refers to the first (second) array in both subfigures. In the absence of mechanical coupling (left), the two arrays synchronize, indicated by the same slope of all phases and the convergence of the phase velocities to horizontal lines. At intermediate mechanical coupling strengths (middle) the oscillators in the second array are synchronized to one frequency while in the first array the phases diverge and the phase velocities stay distinct. This is a chimera state, where the first array oscillates incoherently while the second array is synchronized. When mechanical coupling dominates (right), the two arrays synchronize to the same frequency.

each array evolving with the same slope, whereas phases of different arrays evolve with different slopes; equivalently, the phase velocities corresponding to different arrays converge to different horizontal lines. For large mechanical coupling, we again find that both arrays synchronize, although this time they both synchronize to the same frequency since all phases share a common slope and the phase velocities collapse to the same horizontal line. However, at intermediate coupling strengths we find that the phases of the first array diverge while the second array remains synchronized. Moreover, we observe that the phase velocities of the first array stay distinct while they converge for the second array. This indicates that there is a stable coexistence of synchronization in one 
array and incoherent oscillation in the other, mediated by the mechanical coupling of the arrays.

\section{CONTINUUM LIMIT}

In the regime of self-sustained oscillations, the light field can be adiabatically eliminated and the radiation pressure force $F_{\mathrm{opt}}^{\theta}(t)$ is then a periodic function with fundamental frequency $\bar{\Omega}$. Note that we can neglect the harmonics of the radiation pressure force and approximate $F_{\mathrm{opt}}^{\theta}(t) \approx F_{0} \sin (\bar{\Omega} t+$ $\left.\tilde{\phi}^{\theta}\right)$. By averaging over time and only considering the slow contributions one finds a generalized Kuramoto-like model for the phase evolution $[58,59]$

$$
\begin{aligned}
\dot{\phi}_{i}^{\theta}= & -\Omega_{i}+K_{i} \sin \left(\phi_{i}^{\theta}+\bar{\Omega} t+\tilde{\phi}_{i}^{\theta}\right) \\
& +\sum_{\theta^{\prime}} \sum_{j \in \theta^{\prime}} \frac{\xi_{i j}^{\theta j^{\prime}}}{2} \cos \left(\phi_{j}^{\theta^{\prime}}-\phi_{i}^{\theta}\right) \\
& +\sum_{\theta^{\prime}, \theta^{\prime \prime}} \sum_{\substack{j \in \theta^{\prime \prime} \\
j \in \theta^{\prime}}} \frac{\xi_{i k}^{\theta \theta^{\prime \prime}} \xi_{i j}^{\theta \theta^{\prime}}}{4 \Gamma} \sin \left(\phi_{k}^{\theta^{\prime \prime}}+\phi_{j}^{\theta^{\prime}}-2 \phi_{i}^{\theta}\right) \\
& \left.+\frac{\xi_{i k}^{\theta \theta^{\prime \prime}} \xi_{k j}^{\theta \theta^{\prime}}}{4 \Gamma}\left[\sin \left(2 \phi_{k}^{\theta^{\prime \prime}}-\phi_{j}^{\theta^{\prime}}-\phi_{i}^{\theta}\right)-\sin \left(\phi_{j}^{\theta^{\prime}}-\phi_{i}^{\theta}\right)\right]\right\},
\end{aligned}
$$

where we have defined $K_{i}=F_{0} /\left(2 m \Omega_{i} \tilde{A}_{i}\right)$ and $\xi_{i j}^{\theta \theta^{\prime}}=$ $k_{i j}^{\theta \theta^{\prime}} \tilde{A}_{j} /\left(m \Omega_{i} \tilde{A}_{i}\right)$.

Aiming to obtain analytical insight by using the Kuramotolike model, we follow the analysis of Ref. [14]. Thus we require that the coupling constants $K_{i}$ and $\xi_{i j}^{\theta \theta^{\prime}}$ can be considered global. Performing the continuum limit $N \rightarrow \infty$ requires the conservation of the number of oscillators for consistency. This results in continuity equations for the probability densities $f^{\theta}(\Omega, \phi, t)=g(\Omega) \tilde{f}^{\theta}(\phi, t)$ to find oscillators with natural frequency $\Omega$ to have phase $\phi$ at time $t$ :

$$
\frac{\partial f^{\theta}(\Omega, \phi, t)}{\partial t}+\frac{\partial}{\partial \phi}\left[f^{\theta}(\Omega, \phi, t) v^{\theta}(\Omega, \phi, t)\right]=0 .
$$

Following the method of Ref. [14], we assume (i) a Lorentzian natural frequency distribution $g(\Omega)=\left\{\pi\left[(\Omega-\bar{\Omega})^{2}+\varepsilon^{2}\right]\right\}^{-1}$ and (ii) that the $\tilde{f}^{\theta}(\phi, t)$ are periodic in $\phi$ :

$$
\tilde{f}^{\theta}(\phi, t)=\frac{1}{2 \pi}\left\{1+\left[\sum_{n=1}^{\infty} \tilde{f}_{n}^{\theta}(t) \exp (i n \phi)+\text { c.c. }\right]\right\},
$$

with the Ott-Antonsen property $\tilde{f}_{n}^{\theta}(t)=\left[a_{\theta}(t)\right]^{n}$. This family of probability distributions contains the limiting cases of the uniform distribution for $\tilde{f}_{n}^{\theta}(t)=0$, which signifies no knowledge about the phases, and $\delta(\phi-\Psi)$ for $\tilde{f}_{n}^{\theta}(t)=e^{-i n \Psi}$, signifying perfect synchronization of all phases to $\Psi$. Conveniently, $\tilde{f}^{\theta}(\phi, t)$ converges for all $a_{\theta}(t)=\rho_{\theta} e^{-i \Psi_{\theta}} \in \mathbb{C}$ with $\rho_{\theta} \leqslant 1$ to

$$
\tilde{f}^{\theta}(\phi, t)=\frac{1}{2 \pi} \frac{\left(1-\rho_{\theta}\right)\left(1+\rho_{\theta}\right)}{\left(1-\rho_{\theta}\right)^{2}+4 \rho_{\theta} \sin ^{2}\left[\frac{1}{2}\left(\phi-\Psi_{\theta}\right)\right]} .
$$

To satisfy Eq. (2), the $a_{\theta}(t)$ have to follow a nonlinear evolution (for details, see Appendix C).

In absence of mechanical coupling, $\mu=0$, the phases $\Psi_{\theta}$ decouple. The solution to the dynamics is $\Psi_{\theta}=-\left(\bar{\Omega} t+\bar{\phi}^{\theta}\right)$, and the corresponding stable fixed point is $\rho_{\theta}=-\frac{2 \varepsilon}{\Gamma}+$ $\sqrt{1+\left(\frac{2 \varepsilon}{\Gamma}\right)^{2}} \stackrel{\varepsilon \ll \Gamma}{\longrightarrow} 1$, i.e., perfect synchronization which is in accordance with the numerical results for the small arrays as depicted in the top and middle row of Fig. 3 and the left column of Fig. 4.

If the mechanical coupling dominates $\mu /(2 m \bar{\Omega}) \gg \varepsilon, \Gamma$, we can describe the dynamics of the system in terms of the phase difference $\Delta \Psi:=\Psi_{1}-\Psi_{2}$. We find the fixed points $\left(\rho_{1}, \rho_{2}, \Delta \Psi\right)=(1,1, n \pi)$ with $n \in \mathbb{Z}$, which describe two cases-either (i) the two arrays synchronize perfectly or (ii) each array synchronizes separately but in antiphase with the other array. The sums in the phase evolution are constant for $\phi_{k}^{\theta}=\Psi_{\theta}$ and $\Delta \Psi=n \pi$. This result generalizes the findings for two optomechanical oscillators in Ref. [58], extending its applicability to two arrays of many oscillators analytically. For few oscillators we find this behavior numerically in the bottom row of Fig. 3 and the right column of Fig. 4.

When all terms are relevant, analytical insight can be gained by assuming that array 1 , without loss of generality, is synchronized $\left(\rho_{1}=1\right)$ and stays synchronized $\left(\dot{\rho}_{1}=0\right)$. We obtain

$$
\rho_{2}=\sqrt{\frac{1-\varepsilon \Gamma(2 m \bar{\Omega} / \mu)^{2}}{\cos (2 \Delta \Psi)}} .
$$

Since $\cos (2 \Delta \Psi) \leqslant 1$ for the relevant cases $(|\Delta \Psi| \ll \pi / 4$ $\bmod \pi)$, we find that $\sqrt{1-\varepsilon \Gamma(2 m \bar{\Omega} / \mu)^{2}} \leqslant \rho_{2} \leqslant 1$. Therefore, when the mechanical coupling dominates the heterogeneity $\left(\mu^{2} \gg 4 \varepsilon \Gamma(m \bar{\Omega})^{2}\right)$ the arrays synchronize in phase or antiphase. Importantly, this also means that if there is no heterogeneity $(\varepsilon=0)$ there can be no chimera states $\left(\rho_{2}=1\right)$ which coincides with our numerical findings for small arrays summarized in Fig. 2 and demonstrated in the middle column of Fig. 4.

To sum up, we find that the analytical results for large arrays contain the essential features of our numerical findings for small arrays. Moreover, these results show that in contrast to breaking the symmetry in the coupling topology, chimera states require heterogeneity to exist in this system.

\section{CONCLUSIONS}

Our investigation shows that highly complex nonlinear classical dynamics emerges in globally coupled stronglydriven optomechanical arrays. The fascinating pattern formation leading to the coexistence of coherence and incoherence in two interacting arrays is found to be enforced by the competition between two synchronization mechanisms and breaking the symmetry of identical oscillators to indistinguishable arrays. Since heterogeneity in the natural frequencies of oscillators in realistic setups is of the order of a few linewidths, the physics we describe is of technological relevance. Our study further shows that this complex behavior is readily accessible to experiments.

In closing, we note that to our knowledge establishing a springlike interaction between optomechanical oscillators based on the optomechanical interaction was shown experimentally for only two oscillators so far [64]. However, optomechanics is not the only mechanism to couple the two 
arrays mechanically. Such interactions can also be introduced by strain coupling if the arrays are connected by a substrate, or sound waves if they are in close proximity to one another. Because of their generality, the effects we describe must be accounted for in systems with multiple nonlinear oscillators in close proximity. If chimera states are to be avoided, our results imply a limit to the packing density of such arrays; if chimera states are to be sought, we have shown that a certain amount of heterogeneity must be present.

\section{ACKNOWLEDGMENTS}

The authors would like to thank O. E. Omel'chenko, D. S. Goldobin, and M. Wolfrum for valuable discussions. This work is supported by the European Union's Horizon 2020 research and innovation programme under Grant Agreement No. 732894 (FET Proactive HOT). We acknowledge funding from the Julian Schwinger Foundation.

\section{APPENDIX A: STUDY OF PARAMETER VARIATIONS}

To study the effect of random perturbations in the optomechanical coupling $G$, the mechanical decay rate $\Gamma$, and the mass $m$ we generated different realizations of the two arrays such that one of the three parameters varies for every oscillator with a given standard deviation $\sigma_{X}$ around the mean value $\bar{X}$. The initial conditions and mean values were chosen to give rise to a chimera state. We used values of $\sigma_{X}$ up to $5 \%$ of the mean value, and checked how many of the realizations still yield chimera states. The resulting behavior is documented in Fig. 5. Additionally, we considered breaking the symmetry between the two indistinguishable arrays by perturbing the mechanical frequencies $\Omega_{i}^{\theta}$ for one of the arrays $(\theta=2)$ by adding a constant shift $\Delta \Omega$ while keeping the frequencies of the other array $(\theta=1)$ unchanged such that $\Omega_{i}^{2}=\Omega_{i}^{1}+\Delta \Omega$. This procedure allows us to verify that the indistinguishability of the two arrays is only required approximately, which is an important consideration for experimental implementations.

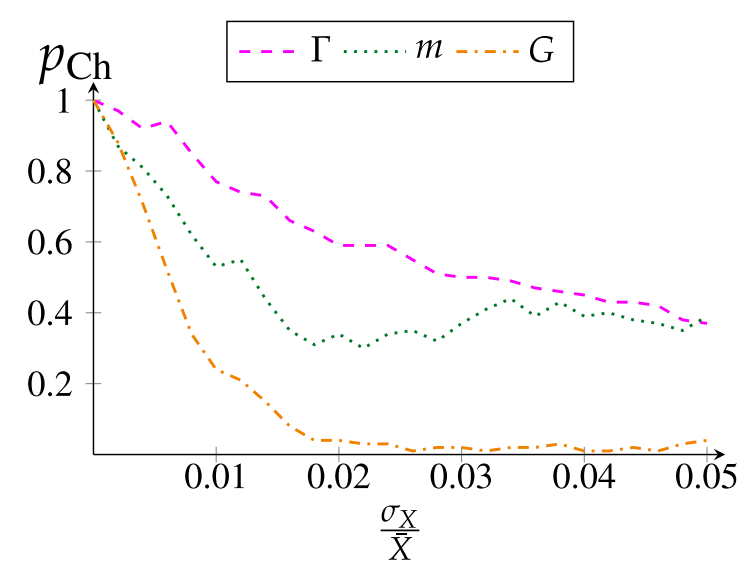

FIG. 5. Stability analysis of chimera states upon variation of the mechanical decay rate $\Gamma$ (dashed), motional mass $m$ (dotted), and optomechanical coupling $G$ (dash dotted). We see that variations on the scale of percent in each parameter still yield a large proportion of chimera states. This indicates that the symmetry of the indistinguishable arrays is only required approximately for an experiment.

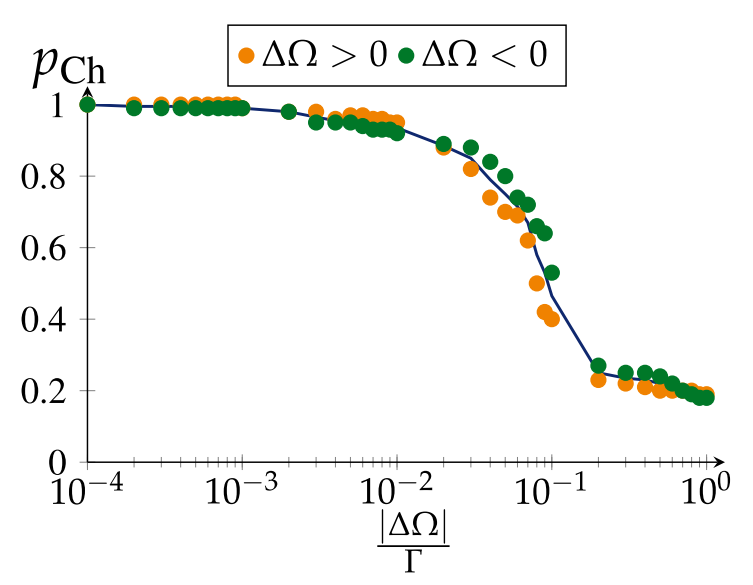

FIG. 6. Stability analysis of chimera states upon variation of the mechanical oscillation frequencies. We see that variations on the scale of a linewidth still result in a significant proportion of chimera states. Thus the symmetry of the indistinguishable arrays can also be broken slightly and still yield chimera states.

The results for variations on the scale of a linewidth $\Gamma$ are depicted in Fig. 6.

\section{APPENDIX B: NUMERICAL ANALYSIS PROCEDURE}

To simulate sample trajectories of our model such as those in Fig. 3, we employ the PYTHON library SCIPY. The numerical algorithm used is the Adams-Moulton method which we employ to evolve random initial conditions with the system parameters indicated in the main text for $0.1 \mathrm{~ms}$ with $4 \times 10^{6}$ steps. We tested convergence of the trajectories for chimera state initial conditions by doubling the steps while keeping the simulation time constant. The phase reconstructions $\phi_{i}^{\theta}$ lead to variations in phase differences $\Delta \phi_{i j}^{\theta}$ of less than $10^{-4} \mathrm{rad}$, which confirms convergence for our purposes.

Our phase reconstruction scheme is based on the analytic signal representation [1] of the time series of the mechanical degrees of freedom $x_{i}^{\theta}(t)$. To specify our problem, we have a real signal $u(t)=x_{i}^{\theta}(t)$ which we want to express as $a(t) \cos [\phi(t)]$ where the real functions $a(t)$ and $\phi(t)$ are the so-called instantaneous amplitude and phase. Equivalently, we want to construct a complex representation $s_{a}(t)=u(t)+$ $i v(t)$ such that $s_{a}(t)=a(t) e^{i \phi(t)}$ holds. There is a unique solution to this problem under the following conditions [67]: (1) the imaginary part $v(t)$ is constructed from $u(t)$; (2) amplitude continuity and differentiability: a continuously differentiable $u(t)$ shall result in a continuously differentiable $a(t)$; (3) phase invariance under scaling: the phase $\phi(t)$ shall be invariant under a scaling of $u(t)$ by a real number; and (4) harmonic correspondence: $u(t)=a_{0} \cos (\omega t+\Phi)$ shall result in $a(t)=a_{0}$ and $\phi(t)=\omega t+\Phi$.

The imaginary part $v(t)$ is then the Hilbert transform of $u(t)$

$$
v(t)=H[u](t)=\frac{1}{\pi} \mathrm{P} . \mathrm{V} . \int_{-\infty}^{\infty} \frac{u(\eta)}{t-\eta} d \eta,
$$

where P.V. denotes the Cauchy principle value. These requirements seem natural, considering that rephrasing the model in terms of differential equations for the amplitude and the phase 
leads to the Kuramoto-like phase evolution. For numerical purposes it is desirable to have sufficient data in the time series in the past and the future. Thus, for all evaluation purposes we discard the final 50000 data points and used the preceding $2^{19}=524288$ samples to compute the Fourier transform and perform phase reconstruction.

\section{APPENDIX C: ANALYTICAL RESULT FOR OTT-ANTONSEN ORDER PARAMETER}

Performing the continuum limit to obtain dynamics for the Ott-Antonsen order parameters requires solving

$$
\frac{\partial f^{\theta}(\Omega, \phi, t)}{\partial t}+\frac{\partial}{\partial \phi}\left(f^{\theta}(\Omega, \phi, t) v^{\theta}(\Omega, \phi, t)\right)=0
$$

for the velocity field $v^{\theta}(\Omega, \phi, t)$ given by

$$
v^{\theta}=-\Omega+K \sin \left(\phi+\tilde{\Omega} t+\tilde{\phi}^{\theta}\right)+\sum_{\theta^{\prime}} \Theta^{\theta^{\prime}} \int_{0}^{2 \pi} d \phi^{\prime} \cos \left(\phi^{\prime}-\phi\right) \tilde{f}^{\theta^{\prime}}\left(\phi^{\prime}, t\right)+\sum_{\theta^{\prime}, \theta^{\prime \prime}} \Xi^{\theta^{\prime \prime}} \Xi^{\theta^{\prime}} \int_{0}^{2 \pi} d \phi^{\prime \prime} \int_{0}^{2 \pi} d \phi^{\prime} T^{\theta^{\prime \prime}, \theta^{\prime}}\left(\phi^{\prime \prime}, \phi^{\prime}, \phi\right),
$$

with the coupling constants $\Theta^{\theta^{\prime}}=N \xi_{i j}^{\theta \theta^{\prime}} / 2$ and $\Xi^{\theta^{\prime}}=\Theta^{\theta^{\prime}} / \sqrt{\Gamma}$, and second-order contributions

$$
\begin{aligned}
T^{\theta, \theta}\left(\phi^{\prime \prime}, \phi^{\prime}, \phi\right)= & \tilde{f}^{\theta}\left(\phi^{\prime \prime}, t\right) \tilde{f}^{\theta}\left(\phi^{\prime}, t\right)\left[\sin \left(2 \phi^{\prime}-\phi^{\prime \prime}-\phi\right)-\sin \left(\phi^{\prime \prime}-\phi\right)+\sin \left(\phi^{\prime \prime}+\phi^{\prime}-2 \phi\right)\right], \\
T^{\theta, \theta}\left(\phi^{\prime \prime}, \phi^{\prime}, \phi\right)= & \tilde{f}^{\theta}\left(\phi^{\prime \prime}, t\right) \tilde{f}^{\theta}\left(\phi^{\prime}, t\right) \sin \left(\phi^{\prime \prime}+\phi^{\prime}-2 \phi\right)-\tilde{f}^{\theta}\left(\phi^{\prime \prime}, t\right) \tilde{f}^{\theta}\left(\phi^{\prime}, t\right)\left[\sin \left(\phi^{\prime \prime}-\phi\right)-\sin \left(2 \phi^{\prime}-\phi^{\prime \prime}-\phi\right)\right], \\
T^{\theta, \theta}\left(\phi^{\prime \prime}, \phi^{\prime}, \phi\right)= & \frac{1}{2}\left\{\tilde{f}^{\theta}\left(\phi^{\prime \prime}, t\right) \tilde{f}^{\theta}\left(\phi^{\prime}, t\right)\left[-\sin \left(\phi^{\prime \prime}-\phi\right)+\sin \left(2 \phi^{\prime \prime}-\phi^{\prime}-\phi\right)\right]+\tilde{f}^{\theta}\left(\phi^{\prime \prime}, t\right) \tilde{f}^{\theta}\left(\phi^{\prime}, t\right) \sin \left(\phi^{\prime \prime}+\phi^{\prime}-2 \phi\right)\right. \\
& \left.+\tilde{f}^{\theta}\left(\phi^{\prime \prime}, t\right) \tilde{f}^{\theta}\left(\phi^{\prime}, t\right)\left[\sin \left(2 \phi^{\prime}-\phi^{\prime \prime}-\phi\right)-\sin \left(\phi^{\prime \prime}-\phi\right)+\sin \left(\phi^{\prime \prime}+\phi^{\prime}-2 \phi\right)\right]\right\}, \text { and } \\
T^{\theta, \theta}\left(\phi^{\prime \prime}, \phi^{\prime}, \phi\right)= & T^{\theta, \theta}\left(\phi^{\prime \prime}, \phi^{\prime}, \phi\right) .
\end{aligned}
$$

To satisfy Eq. (C1), the $a_{\theta}(t)$ have to follow the nonlinear differential equations rewritten in polar coordinates $a_{\theta}(t)=\rho_{\theta} e^{-i \Psi_{\theta}}$ :

$$
\begin{aligned}
\dot{\rho}_{\theta}= & -\varepsilon \rho_{\theta}+\frac{\left(1-\rho_{\theta}^{2}\right)}{2}\left[\frac{\Gamma}{2} \cos \left(\Psi_{\theta}+\tilde{\Omega} t+\tilde{\phi}^{\theta}\right)+\Theta^{\theta} \rho_{\theta} \sin (\Delta \Psi)\right]-\frac{\left(\Xi^{\theta}\right)^{2}}{2} \rho_{\theta}\left(2 \rho_{\theta}^{4}-3 \rho_{\theta}^{2}+1\right) \\
& -\frac{\Xi^{\theta} \Xi^{\theta}}{2} \rho_{\theta}\left[3 \rho_{\theta}^{4}-5 \rho_{\theta}^{2}+2+\rho_{\theta}^{2}\left(\rho_{\theta}^{2}-1\right)\right] \cos (\Delta \Psi)-\frac{\left(\Xi^{\theta}\right)^{2}}{2} \rho_{\theta}\left(2 \rho_{\theta}^{2} \rho_{\theta}^{2} \cos (2 \Delta \Psi)-\rho_{\theta}^{2}-1\right),
\end{aligned}
$$

and

$$
\begin{aligned}
\dot{\Psi}_{\theta}= & -\tilde{\Omega}-\frac{\left(1+\rho_{\theta}^{2}\right)}{2 \rho_{\theta}}\left[\frac{\Gamma}{2} \sin \left(\Psi_{\theta}+\tilde{\Omega} t+\tilde{\phi}^{\theta}\right)-\Theta^{\theta} \rho_{\theta}-\Theta^{\theta} \rho_{\theta} \cos (\Delta \Psi)\right] \\
& -\frac{\Xi^{\theta} \Xi^{\theta} \rho_{\theta}}{2 \rho_{\theta}}\left[\rho_{\theta}^{4}-\rho_{\theta}^{2}-2+\rho_{\theta}^{2}\left(\rho_{\theta}^{2}+1\right)\right] \sin (\Delta \Psi)-\frac{\left(\Xi^{\theta}\right)^{2}}{2} \rho_{\theta}^{2} \rho_{\theta}^{2} \sin (2 \Delta \Psi),
\end{aligned}
$$

where $\Delta \Psi=\Psi_{\theta}-\Psi_{\theta}$. These equations are the basis for the discussion in the main text.

[1] A. Pikovsky, M. Rosenblum, and J. Kurths, Synchronization: A Universal Concept in Nonlinear Sciences (Cambridge University Press, Cambridge, 2003).

[2] Y. Kuramoto, Chemical Oscillations, Waves, and Turbulence (Springer, New York, 1984).

[3] M. Osaka, Appl. Math. 8, 1227 (2017).

[4] D. C. Michaels, E. P. Matyas, and J. Jalife, Circ. Res. 61, 704 (1987).

[5] Z. Jiang and M. McCall, J. Opt. Soc. Am. B 10, 155 (1993).

[6] V. V. Apollonov, S. I. Derzhavin, V. I. Kislov, A. A. Kazakov, Yu. P. Koval', V. V. Kuz'minov, D. A. Mashkovskii, and A. M. Prokhorov, Quantum Electron. 28, 257 (1998).

[7] V. V. Apollonov, Quantum Electron. 44, 102 (2014).

[8] Y. Kuramoto and D. Battogtokh, Nonlinear Phenom. Complex Syst. 5, 380 (2002).
[9] G. Orosz, J. Moehlis, and P. Ashwin, Prog. Theor. Phys. 122, 611 (2009).

[10] D. M. Abrams and S. H. Strogatz, Phys. Rev. Lett. 93, 174102 (2004).

[11] S. Watanabe and S. H. Strogatz, Physica D 74, 197 (1994).

[12] D. M. Abrams, R. Mirollo, S. H. Strogatz, and D. A. Wiley, Phys. Rev. Lett. 101, 084103 (2008).

[13] A. Pikovsky and M. Rosenblum, Phys. Rev. Lett. 101, 264103 (2008).

[14] E. Ott and T. M. Antonsen, Chaos 18, 037113 (2008).

[15] E. Ott and T. M. Antonsen, Chaos 19, 023117 (2009).

[16] M. Wolfrum and O. E. Omel'chenko, Phys. Rev. E 84, 015201(R) (2011).

[17] J. Sieber, O. E. Omel'chenko, and M. Wolfrum, Phys. Rev. Lett. 112, 054102 (2014). 
[18] M. Wolfrum, O. E. Omel'chenko, and J. Sieber, Chaos 25, 015201 (2015).

[19] E. Montbrió, J. Kurths, and B. Blasius, Phys. Rev. E 70, 056125 (2004).

[20] C. R. Laing, Chaos 19, 013113 (2009).

[21] C. R. Laing, Physica D 238, 1569 (2009).

[22] M. Shanahan, Chaos 20, 013108 (2010).

[23] S. Kundu, S. Majhi, B. K. Bera, D. Ghosh, and M. Lakshmanan, Phys. Rev. E 97, 022201 (2018).

[24] C. R. Laing, Chaos 22, 043104 (2012).

[25] I. V. Tyulkina, D. S. Goldobin, L. S. Klimenko, and A. Pikovsky, Phys. Rev. Lett. 120, 264101 (2018).

[26] M. J. Panaggio and D. M. Abrams, Nonlinearity 28, R67 (2015).

[27] O. E. Omel'chenko, Nonlinearity 31, R121 (2018).

[28] M. R. Tinsley, S. Nkomo, and K. Showalter, Nat. Phys. 8, 662 (2012).

[29] L. Schmidt, K. Schönleber, K. Krischer, and V. García-Morales, Chaos 24, 013102 (2014).

[30] K. Schönleber, C. Zensen, A. Heinrich, and K. Krischer, New J. Phys. 16, 063024 (2014).

[31] A. M. Hagerstrom, T. E. Murphy, R. Roy, P. Hövel, I. Omelchenko, and E. Schöll, Nat. Phys. 8, 658 (2012).

[32] J. D. Hart, K. Bansal, T. E. Murphy, and R. Roy, Chaos 26, 094801 (2016).

[33] E. A. Martens, S. Thutupalli, A. Fourrière, and O. Hallatschek, Proc. Natl. Acad. Sci. USA 110, 10563 (2013).

[34] M. H. Matheny, J. Emenheiser, W. Fon, A. Chapman, A. Salova, M. Rohden, J. Li, M. Hudoba de Badyn, M. Pósfai, L. Duenas-Osorio, M. Mesbahi, J. P. Crutchfield, M. C. Cross, R. M. D’Souza, and M. L. Roukes, Science 363, eaav7932 (2019).

[35] M. Aspelmeyer, T. J. Kippenberg, and F. Marquardt, Rev. Mod. Phys. 86, 1391 (2014).

[36] T. J. Kippenberg, H. Rokhsari, T. Carmon, A. Scherer, and K. J. Vahala, Phys. Rev. Lett. 95, 033901 (2005).

[37] F. Marquardt, J. G. E. Harris, and S. M. Girvin, Phys. Rev. Lett. 96, 103901 (2006).

[38] S. Gröblacher, K. Hammerer, M. R. Vanner, and M. Aspelmeyer, Nature (London) 460, 724 (2009).

[39] J. D. Thompson, B. M. Zwickl, F. Marquardt, S. M. Girvin, and J. G. E. Harris, Nature (London) 452, 72 (2008).

[40] J. D. Teufel, T. Donner, D. Li, J. W. Harlow, M. S. Allman, K. Cicak, A. J. Sirois, J. D. Whittaker, K. W. Lehnert, and R. W. Simmonds, Nature (London) 475, 359 (2011).

[41] J. Millen, T. Deesuwan, P. Barkere, and J. Anders, Nat. Nanotechnol. 9, 425 (2014).

[42] U. Delić, M. Reisenbauer, D. Grass, N. Kiesel, V. Vuletić, and M. Aspelmeyer, Phys. Rev. Lett. 122, 123602 (2019).

[43] E. Gil-Santos, C. Baker, A. Lemaître, S. Ducci, C. Gomez, G. Leo, and I. Favero, Nat. Commun. 8, 14267 (2017).

[44] W.-M. Zhang, K.-M. Hu, Z.-K. Peng, and G. Meng, Sensors 15, 26478 (2015).
[45] J. Chan, T. P. M. Alegre, A. H. Safavi-Naeini, J. T. Hill, A. Krause, S. Gröblacher, M. Aspelmeyer, and O. Painter, Nature (London) 478, 89 (2011).

[46] C. F. Ockeloen-Korppi, E. Damskägg, J. M. Pirkkalainen, M. Asjad, A. A. Clerk, F. Massel, M. J. Wooley, and M. A. Sillanpää, Nature (London) 556, 478 (2018).

[47] R. Riedinger, A. Wallucks, I. Marinković, C. Löschnauer, M. Aspelmeyer, S. Hong, and S. Gröblacher, Nature (London) 556, 473 (2018).

[48] N. R. Bernier, L. D. Tóth, A. Koottandavida, M. D. Ioannu, D. Malz, A. Nunnenkamp, A. K. Feofanov, and T. J. Kippenberg, Nat. Commun. 8, 604 (2017).

[49] D. Malz, L. D. Tóth, N. R. Bernier, A. K. Feofanov, T. J. Kippenberg, and A. Nunnenkamp, Phys. Rev. Lett. 120, 023601 (2018).

[50] S. Barzanjeh, M. Wulf, M. Peruzzo, M. Kalaee, P. B. Dieterle, O. Painter, and J. M. Fink, Nat. Commun. 8, 953 (2017).

[51] L. Mercier de Lépinay, E. Damskägg, C. F. OckeloenKorppi, and M. A. Sillanpää, Phys. Rev. Applied 11, 034027 (2019).

[52] A. Xuereb, C. Genes, and A. Dantan, Phys. Rev. Lett. 109, 223601 (2012).

[53] P. Piergentili, L. Catalani, M. Bawaj, S. Zippilli, N. Malossi, R. Natali, D. Vitali, and G. Di Giuseppe, New J. Phys. 20, 083024 (2018).

[54] M. Schmidt, S. Kessler, V. Peano, O. Painter, and F. Marquardt, Optica 2, 635 (2015).

[55] V. Peano, C. Brendel, M. Schmidt, and F. Marquardt, Phys. Rev. X 5, 031011 (2015).

[56] J. P. Mathew, J. del Pino, and E. Verhagen, Nat. Nanotechnol. (2020), doi:10.1038/s41565-019-0630-8.

[57] S. Walter, and F. Marquardt, New J. Phys. 18, 113029 (2016).

[58] G. Heinrich, M. Ludwig, J. Qian, B. Kubala, and F. Marquardt, Phys. Rev. Lett. 107, 043603 (2011).

[59] R. Lauter, C. Brendel, S. J. M. Habraken, and F. Marquardt, Phys. Rev. E 92, 012902 (2015).

[60] R. Lauter, A. Mitra, and F. Marquardt, Phys. Rev. E 96, 012220 (2017).

[61] C. A. Holmes, C. P. Meaney, and G. J. Milburn, Phys. Rev. E 85, 066203 (2012).

[62] N. Lörch, S. E. Nigg, A. Nunnenkamp, R. P. Tiwari, and C. Bruder, Phys. Rev. Lett. 118, 243602 (2017).

[63] E. Amitai, N. Lörch, A. Nunnenkamp, S. Walter, and C. Bruder, Phys. Rev. A 95, 053858 (2017).

[64] M. Zhang, G. S. Wiederhecker, S. Manipatruni, A. Barnard, P. McEuen, and M. Lipson, Phys. Rev. Lett. 109, 233906 (2012).

[65] M. Zhang, S. Shah, J. Cardenas, and M. Lipson, Phys. Rev. Lett. 115, 163902 (2015).

[66] M. F. Colombano, G. Arregui, N. E. Capuj, A. Pitanti, J. Maire, A. Griol, B. Garrido, A. Martinez, C. M. Sotomayor-Torres, and D. Navarro-Urrios, Phys. Rev. Lett. 123, 017402 (2019).

[67] D. Vakman, IEEE Trans. Signal Process. 44, 791 (1996). 